\title{
CHARACTERIZING AMBIGUITY ATTITUDES USING MODEL UNCERTAINTY*
}

\author{
Loï Berger ${ }^{\dagger} \quad$ Valentina Bosetti ${ }^{\ddagger}$
}

\begin{abstract}
We report the results of an experiment eliciting individuals' attitudes toward risk and model uncertainty. Using a joint elicitation procedure, we then precisely quantify the strength of individuals' attitude toward ambiguity in the context of the smooth model and characterize its main properties. Our results provide empirical evidence of decreasing absolute ambiguity aversion (DAAA) and constant relative ambiguity aversion (CRAA). These results shed new light on the way ambiguity attitudes may affect important decisions, such as the choice of health insurance policies or the optimal investment strategy in the face of climate change.
\end{abstract}

Keywords: Ambiguity aversion, Laboratory experiment, Non-expected utility, Subjective probabilities, Decreasing absolute ambiguity aversion

JEL Classification: D81

\footnotetext{
*We thank Mohammed Abdellaoui, Aurélien Baillon, Han Bleichrodt, Itzhak Gilboa, Glenn Harrison, François Pannequin, Massimo Tavoni, Peter Wakker and especially Massimo Marinacci for helpful comments and discussions. We acknowledge audiences at CEAR/MRIC Behavioral Insurance Workshop, the annual seminar of the European Group of Risk \& Insurance Economists (EGRIE), the workshops "Uncertainty and public decision" (EconomiX) and seminar participants at Bocconi University, Rotterdam University, HEC Paris, Fondazione Eni Enrico Mattei, IESEG and EM-Lyon for their helpful comments. We thank Jacopo Crimi, Johannes Emmerling, Michele Peruzzi, Caterina Verrone and Stefano Zenobi for their help with the experiment. This work was supported by the European Union Seventh Framework Programme FP7 [grant agreement no 308329 ADVANCE, ERC-2013-StG 336703-RISICO]; the Agence Nationale de la Recherche [grant ANR-17-CE03-0008-01 INDUCED]; the CASBS. Logistic support from the Bocconi Experimental Laboratory in the Social Sciences (BELSS) for hosting our experimental sessions is appreciated. Some results of this research have previously circulated in a working paper titled "Ellsberg Re-revisited: An Experiment Disentangling Model Uncertainty and Risk Aversion."

${ }^{\dagger}$ CNRS, IESEG School of Management, Univ. Lille, UMR 9221 - LEM, F-59000 Lille, France; and RFF-CMCC European Institute on Economics and the Environment (EIEE), Centro Euro-Mediterraneo sui Cambiamenti Climatici, Italy. E-mail address: l.berger@ieseg.fr.

$\ddagger$ Department of Economics and IGIER, Bocconi University; and and RFF-CMCC European Institute on Economics and the Environment (EIEE), Centro Euro-Mediterraneo sui Cambiamenti Climatici, Italy.
} 
Ambiguity may be high [...] when there are questions of reliability and relevance of information, and particularly where there is conflicting opinion and evidence. (Ellsberg, 1961, p. 659)

\section{Introduction}

Almost sixty years ago, Ellsberg (1961) proposed a series of experiments whose results suggest that people generally prefer situations in which they know the probability of an event occurrence to situations in which this probability is unknown. This behavior, which violates a key axiom of the classic model of choice under uncertainty, is known as ambiguity aversion. It has been the focus of a large body of research that has explored both the theoretical and experimental sides of the topic. According to Ellsberg, this preference is due not to the relative desirability of the possible payoffs, or the relative likelihood of the events affecting them, but to the nature of the information about the relative likelihood of events. In particular, Ellsberg (1961, p. 657) wrote:

What is at issue might be called the ambiguity of this information, a quality depending on the amount, type, reliability and "unanimity" of information, and giving rise to one's degree of "confidence" in an estimation of relative likelihoods.

Ambiguity aversion thus results from a preference for situations in which all events relevant to decision-making are associated with obvious probability assignments, in which everyone agrees (i.e. what is generally called "risk" in economics) to situations in which some events do not have an obvious, unanimously agreeable probability assignment (Ghirardato, 2004).

Ambiguity occurs in virtually all real-life situations. It plays a major role in many problems policymakers and public authorities may face and thus directly affects their decision-making processes. Ambiguity may, for example, result from different opinions of experts about the probability of a particular event (Cabantous, 2007; Cabantous et al., 2011). It may also arise from multiple plausible models predicting the probabilistic effects of alternative policies.

One area in which ambiguity frequently occurs is in health insurance (Gilboa and Marinacci, 2013; Marinacci, 2015). Consider, for example, the problem of a decision maker (DM) who must choose whether to buy insurance against the risk of a heart disease. By browsing the Internet and providing some personal information (e.g., age, cholesterol level, blood pressure, smoking habits), the DM is confronted with different probability assessments of heart disease provided by 
the risk calculators of different hospitals. Thus, different "experts" ${ }^{1}$ provide different probability models for the same event (i.e. suffering a heart attack in the next years). As Gilboa and Marinacci (2013) illustrate, the estimates may vary substantially from one expert to the other. The reason is that simple relative frequencies cannot be used as a definition of the probability of a heart disease because no two human bodies are perfectly identical. Instead, the calculators assess the probabilities for different individuals depending on their characteristics using more complex methods. As a consequence, the assessed probabilities are not perfectly objective: the team in charge of the calculator in each hospital must choose the individual characteristics to be used, the database, and the estimation technique. Together, this results in a set of possible probability models rather than a unique probability distribution.

Another example of ambiguity is environmental disasters for which policymakers may need to design an investment strategy, entailing both mitigation and adaptation efforts in response to climate change. ${ }^{2}$ In this context, different probability distributions may exist to characterize the same event, such as temperature response to cumulated emissions (Heal and Millner, 2014). This happens because there is not enough information available; because different climate predictions exist based on different data sets, different estimation techniques, or numerical models exist; or because different experts provide different probabilities assessments for the same occurrence. As a consequence, little is known about how, for example, global warming will affect the probability of a climate catastrophe (Lenton et al., 2008). As these events have not been encountered by humankind before, their likelihood of occurrence is extremely difficult to quantify precisely. Decisions are therefore generally made based on the advice of scientific experts (e.g., Intergovernmental Panel on Climate Change) whose probability assessments may vary significantly (Kriegler et al., 2009; Berger et al., 2017). In this case as well, different probability models characterizing the same event exist.

Whether regarding health-related issues or environmental questions, what is common to these examples is the idea that ambiguity and elements of insurance of different types are tightly intertwined. Specifically, while the first example entails the provision of market insurance in the presence of ambiguity, the second example

\footnotetext{
${ }^{1}$ Throughout the paper, we refer to experts as individuals or entities who presumably have more information and/or expertise than the DM and who are acting as advisers by providing information (Budescu et al., 2003).

${ }^{2}$ Adaptation is the process of "adjustment to actual or expected climate and its effects. In human systems, adaptation seeks to moderate harm or exploit beneficial opportunities" (IPCC, 2014a), while mitigation is "a human intervention to reduce the sources or enhance the sinks of greenhouse gases" (IPCC, 2014b).
} 
deals with ambiguity and self-insurance (SI)/self-protection (SP) in the sense of Ehrlich and Becker (1972). These risk management tools are generally used to deal with the risk of a monetary loss when market insurance is not available. In both situations, the DM has the opportunity to undertake an effort to modify the distribution of a potential negative outcome (loss). In the case of SI, the effort is intended to reduce the size of the loss, while in the case of SP, the effort aims to reduce the probability of suffering from the loss. In this sense, investments in adaptation efforts in the face of climate change represent an example of SI, while mitigation efforts correspond to SP (Berger, 2016).

To what extent do ambiguity preferences affect the decisions to purchase health insurance policies or to undertake SI/SP efforts in the presence of climate change? The answer to this question fundamentally depends on the strength of ambiguity aversion, as well as on some behavioral properties of the function characterizing ambiguity preferences. In theoretical works, using single-period models, Snow (2011) and Alary et al. (2013) show that ambiguity aversion increases the insurance coverage rate and the optimal level of SI, while it may increase or decrease the optimal level of SP. In a two-period setting, Berger (2016) and Berger et al. (2017) demonstrate that ambiguity aversion alone is not sufficient to sign the effect ambiguity has on the decision to (self-)insure or self-protect. An additional condition of non-increasing absolute ambiguity aversion is also required in most situations in which the degree of model disagreement among experts decreases with the effort. Yet, at an empirical level, the question has not been tackled in much detail.

The aim of this paper is to provide a methodological contribution to this literature. Specifically, what we propose herein is an experimental method capable of quantifying more precisely the extent to which ambiguity aversion exists and characterizing its main properties. ${ }^{3}$ To do so, we decompose ambiguity into two distinct layers of analysis, following the approach of Hansen (2014), Marinacci (2015) and Hansen and Marinacci (2016). The first layer, commonly referred to as risk (or aleatory uncertainty), features the probability measure associated with the randomness of an event. It refers to the physical quantification of uncertainty by means of a probability model that fully specifies the outcome probabilities. This layer of risk within a model is the one typically studied by economists in problems involving decision-making under uncertainty (in the context of SI-SP, see for example Dionne and Eeckhoudt, 1985; Jullien et al., 1999; Eeckhoudt and

\footnotetext{
${ }^{3}$ Few studies have actually attempted to quantify the strength of the ambiguity aversion effect. Notable exceptions, using various models, are those of Abdellaoui et al. (2011); Ahn et al. (2014); Dimmock et al. (2016).
} 
Gollier, 2005). The second layer, referred to as model uncertainty (or epistemic uncertainty), represents uncertainty about which alternative probability model should be used to assign probabilities. ${ }^{4}$ This layer of analysis occurs when the correct risk is itself unknown. This is the case, for example, when multiple expert assessments seem plausible in describing the randomness of a phenomenon.

In a controlled experimental environment that extends Holt and Laury's (2002) and Andersen et al.'s (2008) setups, we expose our subjects to various types of risk and model uncertainty. Using the distinction between the two, our design enables us to quantify precisely the degree of ambiguity aversion and to assess its main properties by means of both non-parametric statistics and structural econometric analyses of choice patterns.

Two main findings emerge from our analysis. First, subjects tend to be both risk and model uncertainty averse but exhibit stronger aversion to model uncertainty than to risk. This behavioral characteristic is evidence of ambiguity aversion, which is then elicited through a joint estimation procedure. Second, and analogously to what has been previously reported for risk aversion (Holt and Laury, 2002, 2005), we find that model uncertainty aversion is decreasing when considered in absolute terms and increasing when considered in relative terms. In terms of ambiguity attitude, we find evidence of decreasing absolute ambiguity aversion (DAAA) and constant relative ambiguity aversion (CRAA).

\section{Experimental procedures}

This section presents the procedures followed in the experiment. We analyze two types of uncertain situations, represented by urns filled with balls that are either red or black. The first situation is a simple risk situation in which the number of red and black balls -and therefore the probability model- is objectively known. The second situation is a model uncertainty situation in which ignorance is first achieved by allowing both the number and the color of the balls in the urn to be unknown before two experts provide their assessment -or probability models- of the urn composition. In our experiment, the experts' models always take the form of precise probabilities that are distinct from each other. ${ }^{5}$ This situation presents uncertainty in two layers: two possible models (the two experts' assessments) rep-

\footnotetext{
${ }^{4}$ The term "epistemic" derives from the Ancient Greek word غ̇ंı edge", while the term "aleatory", which originates from the Latin alea, refers to any game of chance involving dice.

${ }^{5}$ As Gajdos and Vergnaud (2013) note, it is not irrational to face two experts who provide distinct sets of predictions. In particular, this does not imply the presence of any asymmetry of information.
} 
resenting two distinct risks are provided to the subjects. The experiment consists of a sequence of eight tasks. In each task, subjects are confronted with a series of binary choices, presented in the form of ordered tables (see Holt and Laury, 2002).

\subsection{The design}

Each urn describes a particular type of uncertainty that we distinguish as follows:

- Risk: the proportion of red and black balls in the urn is known;

- Model uncertainty: the proportion of red, black, and the total number of balls in the urn are unknown. However, information is provided by two "experts", both giving their own assessment of the composition of the urn.

The probability of drawing a red (resp. black) ball $P(r)$ (resp. $P(b)$ ) is thus objectively known in the case of risk but not in the case of model uncertainty, in which only information about the possible compositions of the urn is given.

CE tasks The first set of tasks takes the form of certainty equivalent (CE) tasks. In these tasks, subjects make a series of choices between an uncertain prospect and a sure amount of money. The uncertain prospect is represented by a risky lottery in the first CE task and by a situation of model uncertainty in the second CE task. Specifically, by letting $X$ denote the set of monetary outcomes, and $\bar{x}_{p} \underline{x}$ the binary lottery yielding $\bar{x} \geq \underline{x} \in X$ with probability $p$ and $\underline{x} \in X$ otherwise, subjects are tasked with making a series of 10 choices between $\bar{x}_{p} \underline{x}$ and different values of $x \in X$ ordered from $\bar{x}$ to $\underline{x} .{ }^{6}$ In the case of model uncertainty, the lottery $\bar{x}_{p} \underline{x}$ is replaced by the uncertain situation denoted $\bar{x}_{\hat{p}_{1} \hat{p}_{2}} \underline{x}$, in which the subject is only given the two experts' assessed probabilities of winning $\hat{p}_{1}$ and $\hat{p}_{2}$. The design of the CE tasks is standard and in accordance with the literature (see e.g., Abdellaoui et al., 2011). To our knowledge, these tasks have never been used in the context of model uncertainty with experts. The CE tasks enable us to characterize the payoff that would make the subject indifferent between the prospect and the sure amount of money in both situations of risk and model uncertainty. These CE tasks, which are extremely easy to understand from the subjects' perspective, help capture treatment effects and differences in individual preferences. They provide a direct measure of the strength of model uncertainty aversion relative to risk aversion without the need to rely on any specific model of choice. To

\footnotetext{
${ }^{6}$ We used the following values in our $\mathrm{CE}$ tasks: $\bar{x}=25, \underline{x}=4, p=0.5$, and $x \in$ $\{25,18,15,14,13,12,10,8,6,4\}$.
} 
ease comparisons, we only consider the case of dogmatic experts in the CE tasks: each expert's assessment is degenerate in the sense that a probability of $100 \%$ is associated with one of the two outcomes. This enables us to isolate the effect of model uncertainty alone and to compare directly risk and model uncertainty preferences.

Other PL tasks In the other tasks of the experiment, we use a double price list (PL) procedure to jointly elicit risk and model uncertainty attitudes. We design two risk tasks following Holt and Laury's (2002) PL standard procedure, ${ }^{7}$ while in the four remaining uncertain tasks, subjects make a series of choices between risk situations $\bar{x}_{p} \underline{x}$ and situations of model uncertainty $\bar{x}_{\hat{p}_{1} \hat{p}_{2}} \underline{x}$. Given the assumptions of a particular model choice that we describe in Section 3, these tasks help us to estimate, using econometric methods, the values of the curvature parameters of two utility functions.

The randomness device As one of our main goals in the experiment is to characterize the way individuals behave in the presence of model uncertainty, we need to ensure that in the absence of experts' information, subjects are in a situation of ignorance. To mimic the situation of ignorance, we construct the model uncertainty urn in such a way that the total number of balls in the urn is itself unknown and number between 1 and 100. We call this modification of Ellsberg's (1961) canonical example, which reduces the information bias due to the peculiarity of the urn representation, the randomness device. In such a situation, the total number of potential objective models is 3045 , which is the cardinality of the Farey sequence of order 100 (see Berger, 2019). ${ }^{8}$

\subsection{Discussion of the design}

We aimed to develop a design that is simple but also emphasizes the difference between risk and model uncertainty. The design presents a few caveats, which we discuss here.

Elicitation procedure The PL procedure is one of the most commonly employed elicitation methods to represent choices between gambles (Andersen et al.,

\footnotetext{
${ }^{7}$ In each task, subjects are asked to make a series of 10 choices between two options (Option A and Option B), each presenting a different lottery (for the full set of tasks, see the online supplemental Appendix).

${ }^{8} \mathrm{~A}$ Farey sequence of order $N$, denoted $\mathfrak{F}_{N}$, is the ascending series of irreducible fractions between 0 and 1 whose denominators do not exceed $N$ (Hardy et al., 1979).
} 
2006). It is widely considered as an easy-to-explain and transparent procedure that rarely confuses subjects about the incentives to respond truthfully (Harrison and Rutström, 2008). However, it also has possible disadvantages. One of its main drawbacks is that by being able to switch freely between the two options as they progress down the decision tables, subjects may end up making inconsistent choices either by switching more than once or by making reverse choices (Charness et al., 2013). While we recognize that these inconsistent behaviors may be problematic, given that they are difficult to rationalize under standard assumptions on preferences and the estimation technique and inference of risk and model uncertainty attitudes require a unique switching point, we decided not to enforce consistent choices in this experiment. ${ }^{9}$ Rather, we view the presence of such behavior as indicative of subjects failing to understand the instructions correctly or, more generally, of confusion on their part. As a result, we discarded this inconsistent data from the analysis of our results. Another potential disadvantage is that the PL procedure only elicits intervals from the subjects rather than point estimates. In addition, it could induce subjects to pick a response in the middle of the table, independent of their true values (framing effect), or it could be more accurate in eliciting probability weighting than the curvature of the utility function (for an in-depth discussion, see Andersen et al., 2006).

Model misspecification While two experts' assessments were provided in the model uncertainty tasks, these experts were not physically present in the lab during the experiment. Although this situation is close to many real-life situations in which experts' opinions are presented without their physical presence, this feature may have led subjects to downplay the experts' opinions or to partially ignore them. Given our design, we cannot guarantee that subjects were not considering alternative models from the ones given by the experts when making their decisions (i.e. misspecification issues). To minimize this potential bias, we proceeded in two steps. First, we created an initial situation of ignorance using the randomness device discussed in the previous section. This device ensures that the DM has a priori no information whatsoever about the possible probability models. Second, we added the desired structural information by means of expert probability models. To induce the subject to use this information, we gave particular attention to the way we presented the experts and their assessments. ${ }^{10}$ We are confident that

\footnotetext{
${ }^{9}$ Several techniques that enforce consistency in subjects' choices are available in the literature (see e.g., Andersen et al., 2006), but with the major drawback that they may significantly bias the results (Charness et al., 2013).

${ }^{10}$ For that purpose, we specifically mentioned the following in the instructions: "These experts are the best we could find for this situation. They are both experienced and have excellent track
} 
most of our subjects incorporated the information provided when making their choices. We are indeed able to show that subjects' choices monotonically follow the stochastic dominance criteria induced by the changes in assessments provided by the two experts (see Section 4.2.2). Relatedly, it could be argued that the absence of physical experts in the room deceived our subjects, who were then tempted to suspect or mistrust the whole experiment. ${ }^{11}$ Again, we are able to show that this suspicion argument is inconsistent with the data we collected. However, to be on the safe side, we explicitly indicated the following in the instructions read aloud at the beginning of the experiment: "This experiment is about decision-making. There will be no deception in the experiment."

\subsection{Recruitment and administration}

The laboratory experiment took place at Bocconi University (Italy). We recruited 189 subjects through an internal experimental economics recruitment system. Each subject was authorized to participate only once and had to sign up in advance for a particular time slot. The experiment was organized into 12 sessions taking place over four days. Each session lasted approximately 75 minutes and comprised between 13 and 19 subjects. Subjects were provided with paper, pen, and a calculator. A session typically started with silent reading of general instructions, which were printed and provided to each subject in the cubicle to which he or she was assigned. The experimenter then read once more the instructions aloud and made sure everything was clear before the subjects began a computerized training session that introduced them to the concepts of risk, model uncertainty and decision tables. The experiment was then performed on computers, with the order of tasks randomized. Overall, the different tasks constituting our experiment were associated with a random incentive system to determine the

records".

${ }^{11}$ While most experimental economists agree that deception should be avoided, nobody knows exactly and with certainty what deception is and is not (Krawczyk et al., 2013). Yet a consensus has emerged across disciplines that deception involves intentional and explicit provision of misinformation (Hertwig and Ortmann, 2008). For example, Adair et al. (1985) define deception as "the provision of information that actively misled subjects regarding some aspect of the study", while Menges (1973) talks about instances in which "the subject is given misleading or erroneous information". The main argument against the use of deception is that it jeopardizes future experiments if the subjects ever find out that they were deceived and report this information to their friends. Banning deception therefore ensures that negative reputational spillover effects are avoided and maintains a "reputation among the student population for honesty in order to ensure that subject actions are motivated by the induced monetary rewards rather than by psychological reactions to suspected manipulation" (Davis and Holt, 1993). In this sense, our design was not subject to deception. In practice indeed, the way we constructed the model uncertainty urn is by randomly selecting one of the two models introduced by the experts in the design, and implementing her assessment. 
final payoff. After all subjects had answered all the questions, they filled out a short socio-economic questionnaire before being told their payoffs (i.e. which of their decisions had been randomly selected, what was the color of the ball drawn from the urn they chose (if any), and what was the corresponding amount they won). Subjects were then paid in cash a $€ 5$ participation fee and the additional amount (up to $€ 35$ ) won depending on the choices they made. The average gain was approximately $€ 18.50$ per subject. We conducted the lab experiment using the experiment software z-Tree (Fischbacher, 2007). Details of the experimental procedure and instructions are available in the online Supplemental Material.

\section{Theoretical predictions}

In this section, we present our theoretical predictions. We use a specific model of choice proposed by Marinacci (2015) building on the work of Klibanoff et al. (2005). This model, known as the smooth ambiguity model, is fairly general. It enriches many of the recent theories of choice under uncertainty in cases in which information is incorporated in the decision problem, while distinguishing between the notions of aleatory and epistemic uncertainty.

\subsection{The smooth ambiguity model}

The DM evaluates acts $f$ whose outcome depends on the realization of an observable state. For each decision, the state may be associated with the color $c \in\{r, b\}$ of the ball (red $r$ or black $b$ ) drawn from the urn under consideration. In other words, each draw represents the realization of a random variable that is characterized by an objective probability distribution, or model, corresponding to a specific composition of the urn. For a given model, the uncertainty about the outcome is of the aleatory type and is generally called risk in economics. ${ }^{12}$ Conditional on the model, the probability of drawing a red (black) ball is therefore objective and refers to a physical concept (in this case, the composition of the urn).

As in many real-life decision problems, however, multiple possible compositions of the urn may exist, and the DM is uncertain about which is the correct one. In this case, the probability model generating the observations is itself uncertain. Following the ex ante information, the DM is able to posit a set of potential models describing the likelihoods of the different states. We assume that this set $M$ of possible compositions of the urn is consistent with the available information

\footnotetext{
${ }^{12}$ Other denominations such as physical, objective, inside, or measurable uncertainty are also present in the literature. All refer to situations in which the probability distribution is known.
} 
provided by the experts. In accordance with Wald (1950), we take this as a datum of the decision problem. ${ }^{13}$ In other words, the DM behaves as if she knows that states are generated by a probability model that belongs to the collection $M$. This therefore means that a second layer of uncertainty may add to the first layer of risk. This additional layer is however not of the aleatory type. Instead, it is of an epistemic nature: the DM does not know which is the most accurate model, and the probability that may be attached to each of them is therefore nothing but a representation of the DM's degrees of belief. Together, this makes the situation uncertain (or ambiguous) rather than risky. We refer to this situation as model uncertainty. The smooth representation of preferences under model uncertainty assumes that epistemic uncertainty is quantified through a single prior probability measure over the set $M$ of models (Marinacci, 2015). The different possible models, which can be fully characterized by their probability of drawing a red ball, are indexed by a parameter $\theta$ and noted by $P_{\theta}(r)$. The DM attaches a probability $\mu(\theta)$ to each, reflecting the personal information she has on their likelihood. ${ }^{14}$ The DM then chooses the act that maximizes her utility given by the two-stage criterion:

$$
U(f)=\mathrm{E}_{\theta}\left(v \circ u^{-1}\right)\left(\sum_{c \in\{r, b\}} \tilde{P}_{\theta}(c) u(f(c))\right) .
$$

In this expression, $\mathrm{E}_{\theta}$ is the expectation operator taken over the prior distribution (i.e. $\mathrm{E}_{\theta} \tilde{X}_{\theta}=\int_{M} X(\theta) d \mu(\theta)$ ), $u$ is the von Neumann and Morgenstern (1944) utility function capturing the DM's risk attitude (i.e. toward aleatory uncertainty), and $v$ captures the attitude toward model uncertainty (i.e. toward epistemic uncertainty). These functions are assumed to be strictly increasing, continuous, and cardinally unique. Criterion (1) may be interpreted as follows: in the first stage, the DM evaluates the expected payoff per each possible model $P_{\theta}(r)$ and expresses it in monetary terms through a CE $c_{\theta} \equiv u^{-1}\left(\sum_{c \in\{r, b\}} \tilde{P}_{\theta}(c) u(f(c))\right)$. These CEs $c_{\theta}$ represent the amount of money that would make the DM indifferent between receiving such amount for sure and facing the risk associated with the model. One $\mathrm{CE} c_{\theta}$ may be computed for each model. It only depends on risk attitude through the function $u$ : the more risk averse the DM is, the lower is $c_{\theta}$. In the second stage, the DM addresses model uncertainty and summarizes the utility of the act by evaluating a global expected payoff $\mathrm{E}_{\theta} v\left(\tilde{c}_{\theta}\right)$ across the CEs in line with her

\footnotetext{
${ }^{13}$ This set of models is analogous to what Ellsberg (1961) calls "reasonable" distributions in his subjective setting. In general, the set $M$ is non-singleton, and the true model is assumed to belong to $M$ (no misspecification issues).

${ }^{14}$ In this sense, the prior probability measure $\mu$ taken over the set of possible models is subjective: it reflects "personal information on models that the DM may have, in addition to objective information $M$ " (Cerreia-Vioglio et al., 2013, p. 6754).
} 
attitude toward model uncertainty $v$ and prior belief $\mu$. Note that in the case in which both attitudes toward the different types of uncertainty are identical (i.e. whenever $v$ is equal -up to an affine transformation- to $u$ ), we recover the classical subjective expected utility model of Cerreia-Vioglio et al. (2013), which encompasses Savage's (1954) model. Representation (1) therefore encompasses both the Savagean subjective expected utility and the classical von Neumann-Morgernstern (when $M$ is a singleton) representations. When attitudes toward risk and model uncertainty are different, representation (1) consists of a version of smooth ambiguity aversion (Klibanoff et al., 2005) in which the ambiguity aversion function is recovered by letting $\phi \equiv v \circ u^{-1}$. In this sense, the DM is ambiguity averse if she is more averse to model uncertainty than to risk (i.e. $v$ more concave than $u$ ).

\subsection{Predictions}

We now present the theoretical predictions for individual choices under risk and model uncertainty. We are particularly interested in characterizing the properties of the two functions $u$ and $v$ representing attitudes toward different types of uncertainty and in relating the smooth ambiguity model uncertainty framework to previous results obtained in the literature.

Hypothesis 1. We expect subjects to be both risk and model uncertainty averse, in the sense that they will generally prefer the degenerate lottery, giving $\sum_{c \in\{r, b\}} \bar{P}(c) f(c)$ with certainty, to any uncertain situation in which an act $f$ yields $f(c)$ with (expected) probability $\bar{P}(c) \forall c \in\{r, b\}$. By letting $C^{R}$ and $C^{M U}$ denote the CEs under risk and model uncertainty, respectively defined as

$$
\begin{aligned}
C^{R} & \equiv u^{-1}\left(\sum_{c \in\{r, b\}} P(c) f(c)\right), \text { and } \\
C^{M U} & \equiv v^{-1}\left(\mathrm{E}_{\theta}\left(v \circ u^{-1}\right)\left(\sum_{c \in\{r, b\}} \tilde{P}_{\theta}(c) u(f(c))\right)\right),
\end{aligned}
$$

and by letting $\bar{C}$ be the sure amount corresponding to the expected gain of the prospect, we expect to observe:

$$
\begin{aligned}
& \bar{C} \geq C^{R} \\
& \bar{C} \geq C^{M U} .
\end{aligned}
$$


Expressed in terms of functional properties, these equations simply become $u^{\prime \prime} \leq 0$ and $v^{\prime \prime} \leq 0$. While Hypothesis 1 is trivial and has been extensively documented in the literature (Holt and Laury, 2002, 2005; Andersen et al., 2008), it is useful for our test of whether ambiguity aversion may result from a stronger aversion to model uncertainty than to risk.

Hypothesis 2. Considering the decomposition of ambiguity into risk and model uncertainty, we expect subjects to prefer risk to model uncertainty, in accordance with the results of Ellsberg (1961). Furthermore, we predict that the degree of model uncertainty aversion (and of ambiguity aversion) will be finite. In other words, subjects will not behave according to a maxmin decision criterion in the sense of Wald (1950). In terms of CEs, we can write these predictions (under the assumption of equal expected values) as:

$$
C^{R} \geq C^{M U}>\underline{C}
$$

where $\underline{C}$ refers to the $\mathrm{CE}$ obtained under the worst possible model. In terms of functions $u$ and $v$, the first part of (6) simply means that $v$ is more concave than $u$, in the sense of Arrow-Pratt (i.e. $-\frac{v^{\prime \prime}}{v^{\prime}} \geq-\frac{u^{\prime \prime}}{u^{\prime}}$ ), while the second part translates to $-\frac{v^{\prime \prime}}{v^{\prime}}<\infty .{ }^{15}$

Hypothesis 3. In line with what is widely accepted in the risk theory literature, and given the similarity of our procedure to that used in Holt and Laury (2002, 2005), we expect to observe decreasing absolute risk aversion (DARA) and increasing relative risk aversion (IRRA) for both functions $u$ and $v{ }^{16}$ Specifically, by changing the values of the gains proposed and the probabilities that are associated with these gains, we expect to observe:

$$
\frac{\partial}{\partial w_{0}}\left[-\frac{u^{\prime \prime}\left(w_{0}\right)}{u^{\prime}\left(w_{0}\right)}\right] \leq 0 \text { and } \frac{\partial}{\partial w_{0}}\left[-\frac{u^{\prime \prime}\left(w_{0}\right)}{u^{\prime}\left(w_{0}\right)} w_{0}\right] \geq 0
$$

where $w_{0}$ denotes the individual's wealth level, which is composed of the individual's background wealth $\omega$ and the expected gain in each lottery $\bar{x}_{p} \underline{x}: w_{0}=$

\footnotetext{
${ }^{15} \mathrm{Or}, \quad \quad \quad \quad v^{-1}\left(\mathrm{E}_{\theta}\left(v \circ u^{-1}\right)\left(\sum_{c \in\left\{r_{i}, b_{i}\right\}} \tilde{P}_{\theta}(c) u\left(f_{i}(c)\right)\right)\right)$

$u^{-1}\left(\min _{\theta} \sum_{c \in\left\{r_{i}, b_{i}\right\}} P_{\theta}(c) u\left(f_{i}(c)\right)\right)$.

${ }^{16}$ To be completely precise, we should discuss "decreasing absolute model uncertainty aversion" and "increasing relative model uncertainty aversion" in the case of function $v$, but for the sake of simplicity we refer to the widely used acronyms DARA and IRRA for the $v$ function as well. While the DARA property is well accepted in the literature, the IRRA property is subject to debate when investigated outside the lab environment (Harrison et al., 2007; Brunnermeier and Nagel, 2008; Chiappori and Paiella, 2011).
} 
$\omega+p \bar{x}+(1-p) \underline{x}$. Similarly, we translate the DARA and IRRA properties of function $v$ to :

$$
\frac{\partial}{\partial w_{1}}\left[-\frac{v^{\prime \prime}\left(w_{1}\right)}{v^{\prime}\left(w_{1}\right)}\right] \leq 0 \text { and } \frac{\partial}{\partial w_{1}}\left[-\frac{v^{\prime \prime}\left(w_{1}\right)}{v^{\prime}\left(w_{1}\right)} w_{1}\right] \geq 0,
$$

where the individual's wealth level $w_{1}$, in situations of model uncertainty, is an average of CE wealth levels under the two experts' models. ${ }^{17}$

Hypothesis 4. Because ambiguity aversion in this setup results from the combination of attitudes toward risk and model uncertainty, we can indirectly characterize the properties of the ambiguity function $\phi$. In particular, we are interested in knowing whether the absolute ambiguity aversion is constant or whether it is increasing or decreasing, in the sense that agents are willing to pay more or less to remove all source of uncertainty as their level of expected utility increases. Constant absolute ambiguity aversion (CAAA), as argued by Grant and Polak (2013), is an implicit characteristic of many of the ambiguity models proposed in the theoretical literature. It is for example implicitly assumed in the models of Gilboa and Schmeidler (1989); Hansen and Sargent (2001) and Maccheroni et al. (2006). By contrast, DAAA is a condition that plays an important role in the determination of the precautionary saving motive under ambiguity (Gierlinger and Gollier, 2008; Berger, 2014), in the chances of survival of ambiguity-averse investors (Guerdjikova and Sciubba, 2015) or in the choice of optimal abatement policies under scientific uncertainty (Berger et al., 2017). We therefore expect to observe

$$
\frac{\partial}{\partial U}\left[-\frac{\phi^{\prime \prime}(U)}{\phi^{\prime}(U)}\right] \leq 0
$$

where $U$ is the individual's expected utility level when the probabilities given by experts are averaged. ${ }^{18}$ (Note that the ambiguity function $\phi \equiv v \circ u^{-1}$ is defined over expected utilities, while $u$ and $v$ are defined over monetary outcomes.)

\footnotetext{
${ }^{17}$ More precisely, in the uncertain situation characterized by $\bar{x}_{\hat{p}_{1} \hat{p}_{2}} \underline{x}, w_{1}$ is computed as $w_{1}=$ $\mu(1) u^{-1}\left(\hat{p}_{1} u(\omega+\bar{x})+\left(1-\hat{p}_{1}\right) u(\omega+\underline{x})\right)+\mu(2) u^{-1}\left(\hat{p}_{2} u(\omega+\bar{x})+\left(1-\hat{p}_{2}\right) u(\omega+\underline{x})\right)$. It is then increasing in the background wealth $\omega$, in the payoff outcomes $\bar{o}$ and $\underline{o}$, and in the expert's probabilities $\hat{p}_{1}$ and $\hat{p}_{2}$.

${ }^{18}$ Baillon and Placido (2019) also tested the CAAA and DAAA hypotheses using probability equivalent tasks (which consist of finding the objective probability at which, for a given prize, a subject is indifferent between betting on the ambiguous event and betting on the objective probability; see Dimmock et al., 2016). At the aggregate level, they found evidence supporting our hypothesis: CAAA was systematically rejected in favor of DAAA.
} 


\section{Results}

In this section, we report the results of our experiment. Section 4.1 uses the results of the CE tasks to present a model-free evaluation of attitudes toward model uncertainty in comparison with risk attitudes. Section 4.2 then proposes a parametric estimation of the smooth ambiguity model by proceeding in two steps. Attitudes toward risk and model uncertainty are first elicited using a joint estimation procedure and then combined to obtain an evaluation of ambiguity attitudes. To facilitate the derivation of our results, we use the following two assumptions.

Assumption 1: No model misspecification. The DM only considers the models given by the experts.

We assume that the exogenously given set of possible models $M$ is well-defined and is not misspecified. As such, and in accordance with Marinacci (2015), we take it as a datum of the decision problem. ${ }^{19}$ As already discussed in the presentation of our design (see Section 2.2), we imposed this assumption, even though nothing theoretically prevents our subjects from considering alternative models distinct from those given by the experts when making their decisions. (Yet, as argued previously, we did our best to avoid such a situation.) While this assumption is not strictly necessary when experts are dogmatic, as in the CE tasks (see subsequently and also Berger and Bosetti, 2019), it is required when a specific model of choice under uncertainty is considered. (For experimental evidence on the role of model misspecification, see Aydogan et al., 2018.)

Assumption 2: Symmetry. The DM considers the two experts symmetrically.

Assumption 2 is akin the one generally imposed in Ellsberg's (1961) type of experiments. In our case, it states that DM is indifferent between betting on red and betting on black when confronted with a model uncertainty situation in which the probabilities given by the experts are symmetric (i.e. $P_{1}(c)=1-P_{2}(c)$ for all $c \in\{r, b\})$. The symmetry assumption has been widely used in the literature and empirically validated by Abdellaoui et al. (2011), Chew et al. (2017) and Epstein

\footnotetext{
${ }^{19}$ Marinacci $(2015$, p. 1013), takes the physical information $M$ as a primitive of the decisionmaking problem and notes that "the DM knows that the true model $m$ generating the states belongs to the posited collection $M . "$
} 
and Halevy (2018). The assumption relies on the symmetry of information argument. Given that the information over the two experts is perfectly symmetric (the experts only differentiate themselves by their names of "Expert 1" and "Expert 2" and their assessments of the urn composition), there is no reason a priori to attach more weight to one than the other. The prior distribution over the models we use reflects this symmetry, such that we have $\mu(1)=\mu(2)=1 / 2$.

\subsection{General results}

The results from the $\mathrm{CE}$ tasks are general in that they characterize attitudes toward risk and model uncertainty directly from the choices made by the subjects in a model-free way. These results therefore do not necessarily rely on the smooth representation of preferences that we discussed previously. The CE tasks enable us to obtain a direct measure of the strength of model uncertainty aversion relative to risk aversion. By considering dogmatic experts (i.e. experts who assess a probability $100 \%$ or $0 \%$ of winning), we can isolate the effect of model uncertainty alone from risk aversion and capture directly model uncertainty preferences. ${ }^{20}$ Moreover, as noted previously, our Assumption 1 is not strictly necessary with dogmatic experts, as their presence ensures that the results we obtain for model uncertainty aversion are, at worst, underestimated. ${ }^{21}$

Table 1 reports the descriptive statistics of the CEs associated with the risk and model uncertainty prospects (i.e. $25_{\frac{1}{2}} 4$ and $25_{10} 4$, respectively). We use the decision item at which the subject switches from the sure outcome to the uncertain prospect to approximate her CEs. Formally, for a subject who switches from the sure outcome after line $i \in\{0,1, \ldots, 10\}$ in the decision table, the $\mathrm{CE}, C^{J}$, for $J \in\{R, M U\}$, is given by

$$
C^{J}= \begin{cases}x_{1}+\frac{1}{2}\left(x_{1}-x_{2}\right) & \text { if } \quad i=0 \\ \frac{1}{2}\left(x_{i+1}+x_{i}\right) & \text { if } i \in\{1, \ldots, 9\} \\ x_{10}-\frac{1}{2}\left(x_{9}-x_{10}\right) & \text { if } \quad i=10\end{cases}
$$

where $i=0$ means that the subject chooses the uncertain situation already in the

\footnotetext{
${ }^{20} \mathrm{In}$ the case of model uncertainty with dogmatic experts, the $\mathrm{CE}$ collapses to $C^{M U}=v^{-1}\left(\sum_{c \in\{r, b\}} \frac{1}{2} v(f(c))\right)$, while the $\mathrm{CE}$ of the 50-50 risk situation is $C^{R}=$ $u^{-1}\left(\sum_{c \in\{r, b\}} \frac{1}{2} u(f(c))\right)$.

${ }^{21}$ Indeed, if subjects were to consider any alternative model other than those belonging to the exogenously given set $M=\{0,1\}$, the preferences associated with the observed choices would need to reflect a higher aversion to model uncertainty than what we present in what follows (as any other symmetric distribution consistent with Assumption 2 in the space of expected utilities would consist of a mean-preserving contraction of the dogmatic experts' distribution).
} 
first choice option, and $i=10$ means that she always chooses the sure outcome. The reported CEs therefore correspond to the midpoint between the highest outcome for which the uncertain prospect is preferred and the lowest outcome that is preferred to the uncertain prospect. The consistent sub-sample contains 169 subjects. As Table 1 shows, the results confirm our first two hypotheses: in general,

Table 1: Descriptive Statistics of the CEs

\begin{tabular}{lcccccc}
\hline \hline & Mean & Std. Error & Median & Min & Max & Obs. \\
\hline$C^{R}$ & 12.57 & 0.17 & 13.5 & 7 & 16.5 & 169 \\
$C^{M U}$ & 11.04 & 0.24 & 11 & 3 & 28.5 & 169 \\
\hline
\end{tabular}

subjects are ready to pay a higher premium, measured as the difference between the expected gain and the $\mathrm{CE}\left(\bar{C}-C^{J}\right)$, to avoid a situation characterized by epistemic uncertainty than to avoid a situation characterized by aleatory uncertainty. In particular, for an expected outcome $\bar{C}=14.5$, the mean amount that the subjects deem equivalent to the risk situation is $C^{R}=12.6$, while under model uncertainty, it is $C^{M U}=11$. The difference between the CEs $\left(C^{R}-C^{M U}\right)$ is significantly positive $(p$-value $=8.9 \mathrm{e}-11)$, and both are higher than the $\mathrm{CE}$ obtained under the worst possible model $\underline{C}=4$.

The distribution of $C^{R}$ second order stochastically dominates that of $C^{M U}$. While $C^{R}$ does not first order stochastically dominate (FOSD) $C^{M U}$, this is only because one subject systematically preferred the model uncertainty situation to the sure outcome (even when the choice was made between $€ 25$ for sure and a situation in which one expert expressed a $100 \%$ probability that the gain is $€ 25$, when the other expert expressed a $100 \%$ probability that the gain is $€ 4$ ). We can only speculate what the preferences of this subject are. He or she could be optimistic and always trust the expert predicting the highest outcome. In this sense, the first choice would express indifference between two situations yielding $€ 25$. After we remove this subject from the sample, we recover the result that $C^{R}$ FOSD $C^{M U}$. We illustrate this result in Figure 1 and Table 3, which display the proportions of safe choices, expressed by preference for the sure amount, for each of the 10 decisions in the risk and model uncertainty situations, respectively. The dashed line represents the prediction under the assumption of neutrality (to either risk or model uncertainty). In this case, the CEs of both situations are the same, and the probability that the sure outcome is chosen is 1 for the first three decisions and 0 for the remaining ones. The blue line presents the observed choice frequency of the sure outcome option in the risk situation. As the figure shows, this 
Figure 1: Proportion of safe choices in the CE tasks

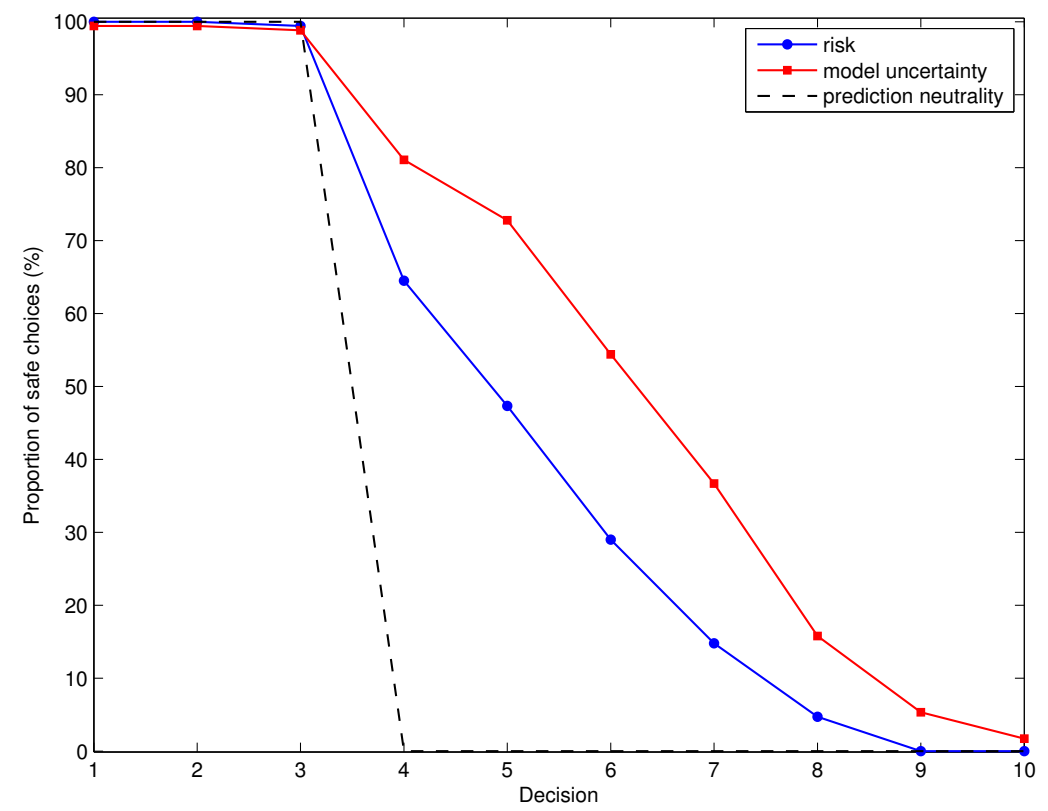

outcome is at the right of the risk-neutral prediction, indicating a tendency for risk aversion among subjects $\left(C^{R} \leq \bar{C}\right)$. The red line represents the observed frequency in the case of model uncertainty and suggests that the subjects manifest a stronger aversion to model uncertainty than to risk $\left(C^{M U} \leq C^{R} \leq \bar{C}\right)$. Finally, the subjects did not express extreme model uncertainty aversion (which would have consisted of a proportion safe choice of $100 \%$ for each decision). Only two subjects (i.e. $1.2 \%$ of the sample) expressed an extreme form of pessimism by systematically selecting the certain outcome when confronted with model uncertainty. ${ }^{22}$ We can therefore confidently reject the maxmin expected utility hypothesis in which subjects only consider the worst possible model (Wald, 1950). A Wilcoxon signedrank test statistically confirms $(p$-value $=3.2 \mathrm{e}-12)$ that the risk alternative is valued differently than the corresponding model uncertainty prospect. ${ }^{23}$

At the individual level, we show, in Table 2, that $52 \%$ (resp. $37 \%, 11 \%$ ) of the sample expressed a stronger (resp. equal, weaker) aversion to model uncertainty than to risk. These proportions' difference is statistically significant. In line with the theoretical model presented previously, these subjects are exhibiting ambiguity aversion, neutrality, and loving, respectively.

\footnotetext{
${ }^{22}$ A looser definition of extreme pessimism, which treats subjects who expressed nine safe choices before switching to the model uncertainty situation as indifferent between the two options, leads to $5.3 \%$ of of individuals with extreme model uncertainty aversion.

${ }^{23}$ The significance of the one-sided test, in which the alternative hypothesis is that the median of the switching point in the model uncertainty task is greater than in the risk aversion task, is $4.2 \mathrm{e}-12$.
} 
Table 2: Comparison between attitudes toward risk and model uncertainty

\begin{tabular}{lcccc}
\hline \hline & $C^{R}<C^{M U}$ & $C^{R}=C^{M U}$ & $C^{R}>C^{M U}$ & Total \\
\hline Count & 19 & 62 & 88 & 169 \\
(Relative frequency) & $(11.2 \%)$ & $(36.7 \%)$ & $(52.1 \%)$ & $(100 \%)$ \\
\hline
\end{tabular}

Anticipating the parametric analysis developed in the next section, Table 3 provides the implied interval for the parameters of risk or model uncertainty aversion in the context of the smooth model. For this exercise, we consider the special case of power (CRRA) ${ }^{24}$ functions. Using an estimation procedure (described

Table 3: Classification of choices (CE tasks)

\begin{tabular}{cccc}
\hline \hline \multirow{2}{*}{$\begin{array}{c}\text { Number } \\
\text { of safe } \\
\text { choices }\end{array}$} & $\begin{array}{c}\text { Range of relative risk or model } \\
\text { uncertainty aversion: } \\
u(x) \text { or } v(x)=x^{1-r} / 1-r\end{array}$ & Proportion of choices \\
\cline { 3 - 4 } & $r<-1.04$ & $\begin{array}{c}\text { Model } \\
\text { Uncertainty }\end{array}$ \\
\hline $0-1$ & $-1.04<r<-0.12$ & $0.00 \%$ & $0.59 \%$ \\
2 & $-0.12<r<0.12$ & $34.91 \%$ & $0.59 \%$ \\
3 & $0.12<r<0.34$ & $17.16 \%$ & $8.75 \%$ \\
4 & $0.34<r<0.55$ & $18.34 \%$ & $15.38 \%$ \\
5 & $0.55<r<1$ & $14.20 \%$ & $20.71 \%$ \\
6 & $1<r<1.55$ & $10.06 \%$ & $21.89 \%$ \\
7 & $1.55<r<2.58$ & $4.73 \%$ & $9.47 \%$ \\
8 & $2.58<r$ & $0.00 \%$ & $5.33 \%$ \\
$9-10$ & & & \\
\hline
\end{tabular}

next), we also find the best estimates for the coefficients of relative risk and model uncertainty aversion when both $u$ and $v$ are of a CRRA type to be respectively $r_{u}=0.42$ and $r_{v}=0.83$ in the CE tasks.

\subsection{Characterizing smooth preferences}

In this section, we use the choices made in the CE and PL tasks to further characterize preferences under uncertainty in the context of the smooth ambiguity model. In particular, we use the 80 binary choices each subject typically provided to infer attitudes toward risk and model uncertainty, and then we use this information to quantify the degree of ambiguity aversion. In total, $14 \%$ of the choices made in the eight PL tasks were deemed inconsistent (reverse choices or multiple

\footnotetext{
${ }^{24} \mathrm{~A}$ utility function has the CRRA property if it takes the form $u(x)=\frac{x^{1-r}}{1-r}$, where $r$ is the coefficient of relative risk aversion (when $r=1$, this collapses to $u(x)=\ln x$ ).
} 
switching points) and were discarded from the analysis. This is in line with what has previously been reported in other experiments (Holt and Laury, 2002).

\subsubsection{Preliminary comment}

As the smooth ambiguity model we test involves two distinct behavioral characteristics (i.e. risk and model uncertainty attitudes), the experimental procedure must be designed such that it generates data that are rich enough to disentangle the different components and ultimately allow for estimating the subjects' attitude toward ambiguity. The double PL procedure, which presents choices in the presence of both aleatory and epistemic uncertainty, is designed for this purpose. It enables us to jointly elicit risk and model uncertainty attitudes. To understand the importance of using a joint procedure, consider the identification of risk and model uncertainty under the smooth model. Assuming that expression (1) correctly describes preferences over uncertain alternatives, a subject is indifferent between two options $\bar{x}_{p} \underline{x}$ and $\bar{x}_{\hat{p}_{1} \hat{p}_{2}} \underline{x}$ if and only if:

$$
\begin{aligned}
\left(v \circ u^{-1}\right)(p u(\omega+\bar{x})+(1-p) u(\omega+\underline{x})) & =\frac{1}{2}\left(v \circ u^{-1}\right)\left(\hat{p}_{1} u(\omega+\bar{x})+\left(1-\hat{p}_{1}\right) u(\omega+\underline{x})\right) \\
& +\frac{1}{2}\left(v \circ u^{-1}\right)\left(\hat{p}_{2} u(\omega+\bar{x})+\left(1-\hat{p}_{2}\right) u(\omega+\underline{x})\right),
\end{aligned}
$$

where $\omega$ represents background wealth. When considered in terms of attitude toward ambiguity, the identity $\phi \equiv v \circ u^{-1}$ enables us to rewrite (11) as

$$
\begin{aligned}
\phi(p u(\omega+\bar{x})+(1-p) u(\omega+\underline{x})) & =\frac{1}{2} \phi\left(\hat{p}_{1} u(\omega+\bar{x})+\left(1-\hat{p}_{1}\right) u(\omega+\underline{x})\right) \\
& +\frac{1}{2} \phi\left(\hat{p}_{2} u(\omega+\bar{x})+\left(1-\hat{p}_{2}\right) u(\omega+\underline{x})\right) .
\end{aligned}
$$

From (11) and (12), it is clear that estimating model uncertainty aversion $(v)$ or ambiguity aversion $(\phi)$ under the assumption of risk neutrality ( $u$ linear) will yield the same result. Ambiguity aversion is therefore significantly overestimated when risk neutrality is assumed. ${ }^{25}$ If we relax the assumption of risk neutrality and let risk aversion $-u^{\prime \prime} / u^{\prime}$ be different than zero, it becomes clear from the relationship $-\phi^{\prime \prime} / \phi^{\prime}=\left(-v^{\prime \prime} / v^{\prime}+u^{\prime \prime} / u^{\prime}\right) / u^{\prime}$ that the implied degree of absolute ambiguity aversion is lower. Therefore, we cannot capture the distinction between model uncertainty and ambiguity aversion without estimating the level of risk aversion, for which separated risk tasks also need to be performed.

\footnotetext{
${ }^{25}$ We discuss the estimation results of this particular case in the Appendix.
} 


\subsubsection{The double PL tasks}

In the double PL tasks, we exploit the comparisons between risk and model uncertainty prospects with experts that are no longer dogmatic. The purely risk tasks are based on Holt and Laury's (2002) mechanism, which has become a standard for elicitation of risk aversion. The model uncertainty tasks are constructed analogously. Table 4 illustrates the type of choices proposed in this part of the experiment. In this example, Option A offers either $€ 35$ or $€ 1$ with equal probability, while Option B offers the same outcomes with unknown probabilities. In the latter case, respondents are given information in the form of the two experts' assessments. In the first decision for example, Expert 1 assesses the probability of obtaining $€ 35$ to be $50 \%$, while Expert 2 is $100 \%$ sure that the outcome will be $€ 1$. Although we report, in Table 4 , the expected value of Option A $\left(\mathrm{EV}^{\mathrm{A}}\right)$, the expected value of Option B if either Expert 1 or Expert 2 is correct (respectively $\mathrm{EV}_{1}^{\mathrm{B}}$ and $\left.\mathrm{EV}_{2}^{\mathrm{B}}\right)$, the average expected value of Option $\mathrm{B}\left(\mathrm{EV}^{\mathrm{B}}\right)$ under the assumption of equal weights attached to each expert, and its standard deviation $\left(\mathrm{SD}^{\mathrm{B}}\right)$, we did not give these to subjects during the experiment. While the expected value of

Table 4: Payoff table in the model uncertainty aversion tasks

\begin{tabular}{|c|c|c|c|c|c|c|c|c|c|c|c|}
\hline \multicolumn{3}{|c|}{ Option A } & \multicolumn{4}{|c|}{ Option B } & \multirow{2}{*}{$\begin{array}{c}\mathrm{EV}^{\mathrm{A}} \\
(€)\end{array}$} & \multirow{2}{*}{$\begin{array}{c}\mathrm{EV}_{1}^{\mathrm{B}} \\
(€)\end{array}$} & \multirow{2}{*}{$\begin{array}{r}\mathrm{EV}_{2}^{\mathrm{B}} \\
(€)\end{array}$} & \multirow{2}{*}{$\begin{array}{c}\mathrm{EV}^{\mathrm{B}} \\
(€)\end{array}$} & \multirow{2}{*}{$\begin{array}{c}\mathrm{SD}^{\mathrm{B}} \\
(€)\end{array}$} \\
\hline $\bar{x}$ & $p$ & $\underline{x}$ & $\bar{x}$ & $\hat{p}_{1}$ & $\hat{p}_{2}$ & $\underline{x}$ & & & & & \\
\hline 35 & 0.5 & 1 & 35 & 0.5 & 0 & 1 & 18 & 18 & 1.0 & 9.5 & 8.5 \\
\hline 35 & 0.5 & 1 & 35 & 0.9 & 0 & 1 & 18 & 31.6 & 1.0 & 16.3 & 15.3 \\
\hline 35 & 0.5 & 1 & 35 & 0.9 & 0.09 & 1 & 18 & 31.6 & 4.1 & 17.8 & 13.8 \\
\hline 35 & 0.5 & 1 & 35 & 0.8 & 0.19 & 1 & 18 & 28.2 & 7.5 & 17.8 & 10.4 \\
\hline 35 & 0.5 & 1 & 35 & 0.8 & 0.21 & 1 & 18 & 28.2 & 8.1 & 18.2 & 10.0 \\
\hline 35 & 0.5 & 1 & 35 & 0.7 & 0.31 & 1 & 18 & 24.8 & 11.5 & 18.2 & 6.6 \\
\hline 35 & 0.5 & 1 & 35 & 0.6 & 0.41 & 1 & 18 & 21.4 & 14.9 & 18.2 & 3.2 \\
\hline 35 & 0.5 & 1 & 35 & 0.55 & 0.46 & 1 & 18 & 19.7 & 16.6 & 18.2 & 1.5 \\
\hline 35 & 0.5 & 1 & 35 & 0.51 & 0.50 & 1 & 18 & 18.3 & 18.0 & 18.2 & 0.2 \\
\hline 35 & 0.5 & 1 & 35 & 0.61 & 0.60 & 1 & 18 & 21.7 & 21.4 & 21.6 & 0.2 \\
\hline
\end{tabular}

Option A is kept constant throughout the various decisions, the expected value of Option B is increasing when proceeding down the table. By contrast, the standard deviation is decreasing (except between the first and second decision). Overall, we construct the decision table in such a way that, for any increasing utility function, Option B always stochastically dominates (in the first- or second-order sense) the previous decision when moving down the table. ${ }^{26}$ Under the smooth ambiguity

\footnotetext{
${ }^{26}$ We can easily show that Option B in the second decision first order stochastically dominates Option B in the first decision and that Option B in the fourth decision second order stochastically dominates Option B in the third decision.
} 
model, this feature should induce subjects to switch only once, from Option A to Option B, while progressing down the table. The subjects engaged in four tasks, similar to that illustrated in Table 4, that varied in the proposed payoffs and probabilities. For the set of payoffs and probabilities, the final payoffs span the range of income over which we are estimating model uncertainty aversion, which is the same as that over which risk aversion is estimated. ${ }^{27}$

\subsubsection{Eliciting risk and model uncertainty attitudes}

We use each of the subjects' binary choices to estimate the parameters of two latent utility functions that explain these choices. We allow for a stochastic error structure, instead of a strictly deterministic structural estimation procedure, because we want to allow subjects to make some errors and, at the same time, to account for the panel structure of the data. Given the support for the CRRA hypothesis in the empirical literature on risk aversion (Harrison et al., 2007; Brunnermeier and Nagel, 2008; Chiappori and Paiella, 2011) but, at the same time, the experimental evidence found in favor of increasing relative risk aversion (IRRA) (Holt and Laury, 2002), we maintain a generic parametric structure for the identification problem. We let both utility functions representing risk and model uncertainty attitudes be of the expo-power (EP) form (Saha, 1993). In the case of risk, this means that the utility function takes the form:

$$
u(x+\omega)=\frac{1-\exp \left(-a_{u}(\omega+x)^{1-r_{u}}\right)}{a_{u}} .
$$

This representation includes CRRA and CARA (constant absolute risk aversion) as special cases and exhibits the desirable properties of DARA and IRRA for positive values of the parameters $a_{u}$ and $r_{u}$ (Abdellaoui et al., 2007). ${ }^{28}$ Note the presence of $\omega$, representing background wealth, in expression (13). As is generally the case in the experimental literature, we assume $\omega=0$. In a situation in which $\omega>0$, however, the same observed choices would imply higher risk aversion. Using the procedure proposed by Andersen et al. (2008), we then construct the expected utility of the two options constituting each decision by using candidate values of parameters $a_{u}$ and $r_{u}$ and a linking index to infer the likelihood of

\footnotetext{
${ }^{27}$ In particular, Option A in the three other uncertain tasks takes the values: $3.5_{0.5} 0.1,35_{0.1} 1$, and $35_{0.9} 1$, and Option B covers the space around these values analogously to what is presented in Table 4. For a detailed description of our experimental design, see the Online supplemental Appendix.

${ }^{28} \mathrm{As}$ is well known, the Arrow-Pratt index of relative risk aversion of the EP function is $-u^{\prime \prime}(x+\omega)(\omega+x) / u^{\prime}(x+\omega)=r_{u}+a_{u}\left(1-r_{u}\right)(\omega+x)^{1-r_{u}}$. We can then easily show that this function exhibits CRRA of value $r_{u}$ when $a_{u}=0$, and CARA of $a_{u}$ when $r_{u}=0$.
} 
the observed choice. The parameters of the latent utility function (13) are then chosen to maximize the likelihood of obtaining the observed ranking of the different options, taking into account a Luce (1959) error specification with a structural noise parameter. ${ }^{29}$

The first part of Table 5 presents the estimates obtained from the risk tasks. Given the prominent position CRRA has achieved in the theoretical and empirical literature, we provide both the estimates for the cases in which $u$ is of the CRRA and EP type. The estimate of the CRRA parameter we obtain is 0.28 , which is

Table 5: Estimates of risk, model uncertainty, and ambiguity preferences

\begin{tabular}{|c|c|c|c|c|c|c|}
\hline & \multicolumn{2}{|c|}{$u$} & \multicolumn{2}{|c|}{$v$} & \multicolumn{2}{|c|}{$\phi$} \\
\hline & CRRA & $\mathrm{EP}$ & CRRA & $\mathrm{EP}$ & CRAA & $\mathrm{EP}$ \\
\hline$a$ & & $\begin{array}{l}0.0294^{* * *} \\
(0.00215)\end{array}$ & & $\begin{array}{l}0.152^{* * *} \\
(0.0542)\end{array}$ & & $\begin{array}{c}-1.802 \\
(0.9655)\end{array}$ \\
\hline$r$ & $\begin{array}{l}0.279^{* * *} \\
(0.0119)\end{array}$ & $\begin{array}{l}0.135^{* * *} \\
(0.0193)\end{array}$ & $\begin{array}{l}0.738^{* * *} \\
(0.0210)\end{array}$ & $\begin{array}{l}0.467^{* * *} \\
(0.0542)\end{array}$ & $\begin{array}{l}0.534^{* * *} \\
(0.0261)\end{array}$ & $\begin{array}{c}0.86^{* * *} \\
(0.0452)\end{array}$ \\
\hline Noise parameter & $\begin{array}{c}0.103^{* * *} \\
(0.00327)\end{array}$ & $\begin{array}{c}0.105^{* * *} \\
(0.00330)\end{array}$ & $\begin{array}{l}0.0358^{* * *} \\
(0.00237)\end{array}$ & $\begin{array}{l}0.0534^{* * *} \\
(0.00343)\end{array}$ & $\begin{array}{l}0.0476^{* * *} \\
(0.00213)\end{array}$ & $\begin{array}{c}0.0363^{* * *} \\
(0.00184)\end{array}$ \\
\hline Observations & 5320 & 5320 & 7570 & 7570 & 7570 & 7570 \\
\hline Log-likelihood & -1550.3 & -1516.8 & -3682.5 & -3682.1 & -3680.6 & -3675.1 \\
\hline
\end{tabular}

lower than the one we obtain using the CE task only. When the EP specification is considered, we estimate $r_{u}=0.135$ and $a_{u}=0.029$, which implies IRRA. While the focus of our analysis is on comparing these estimates with those obtained for the model uncertainty function $v$, we note that their absolute magnitudes are consistent with the results obtained by Holt and Laury (2002) and Andersen et al. (2008). We however recognize that the estimates we obtain only hold locally over the domain of stakes offered in our experiment. ${ }^{30}$ The last two rows of Table 5 present information about the data used (30 risk choices for each of the 189 subjects, minus the inconsistent choices that are discarded) and the resulting loglikelihood values. As the table shows, the log-likelihood of the EP specification

\footnotetext{
${ }^{29}$ The statistical specification we use allows us to take into account the correlation between responses given by the same subject. Robust estimates considering clustering corrections are available in Appendix B. There is essentially no difference in the significance of our estimates in this case.

${ }^{30}$ The relatively low value of relative risk aversion we obtain in comparison with empirical studies using real data (Attanasio et al., 2002; Chetty, 2006) may be due to the zero or low value of background consumption. When we instead consider background wealth (in the sense of Arrow-Pratt or lifetime wealth), the value of relative risk aversion may become much larger, in line with the Rabin's critique.
} 
is slightly better than the CRRA specification, but this is not surprising given that the estimates are all significant; therefore, the hypothesis $a_{u}=0$ is rejected. Given the superiority of the EP specification in explaining the observed choices in the risk tasks, this is the specification we consider in the remaining part of the estimation procedure. We then estimate the model uncertainty aversion function $v$, which takes the general EP form:

$$
v(C E)=\frac{1-\exp \left(-a_{v}(C E)^{1-r_{v}}\right)}{a_{v}},
$$

where $C E$ represents the $C E$ wealth for a given model $\theta: C E \equiv u^{-1}\left(\hat{p}_{\theta} u(\bar{x})+\right.$ $\left.\left(1-\hat{p}_{\theta}\right) u(\underline{x})\right)$. The second part of Table 5 presents the estimates obtained from our five uncertain PL tasks. We also provide estimates for the special cases of $v$ being of the CRRA type $\left(a_{v}=0\right)$ for indicative purposes. In this case, the coefficient estimated is significantly higher than the one obtained in the case of risk. It should be noted, however, that this specification leads to a smaller loglikelihood value than the general expo-power formulation (14). Focusing on the EP specification, we note that the estimates we obtain $\left(a_{v}=0.152\right.$ and $\left.r_{v}=0.467\right)$ with the joint identification procedure are both significantly positive. This implies that our subjects exhibit both decreasing absolute model uncertainty aversion and increasing relative model uncertainty aversion.

Our interest lies in comparing the estimates obtained for model uncertainty with those obtained for risk. In Figure 2, we provide the paths of estimated absolute and relative aversion indexes for both risk and model uncertainty over the experimental prize domain. As predicted, we show that the indexes are both decreasing in the monetary outcome when considered in absolute terms and increasing in relative terms (DARA and IRRA). We also directly show in Figure 2 that the degree of model uncertainty aversion is significantly higher (in both absolute and relative terms) than that of risk aversion. This result confirms our main hypothesis (Hypothesis 2) that subjects are more averse to model uncertainty than to risk. Specifically, while the index of relative risk aversion is respectively 0.32 and 0.62 when the monetary outcome considered is either $x=10$ or $x=30$, the index of relative model uncertainty aversion takes the values of 0.74 and 0.96 for the corresponding outcomes. (Note that in the special case in which both $u$ and $v$ are of the CRRA type, the indices of relative aversion to risk and model uncertainty are $r_{u}=0.28$ and $r_{v}=0.73$ when jointly estimated.$^{31}$ ) These differences

\footnotetext{
${ }^{31}$ To assess the sensitivity of the model uncertainty aversion index to variations in relative risk aversion, we also used the maximum likelihood procedure to estimate $r_{v}$ using different (exogenously given) values of $r_{u}$. These additional results are available in Appendix C.
} 
Figure 2: Absolute (left) and relative (right) risk and model uncertainty aversion using EP estimates (95\% confidence in gray).
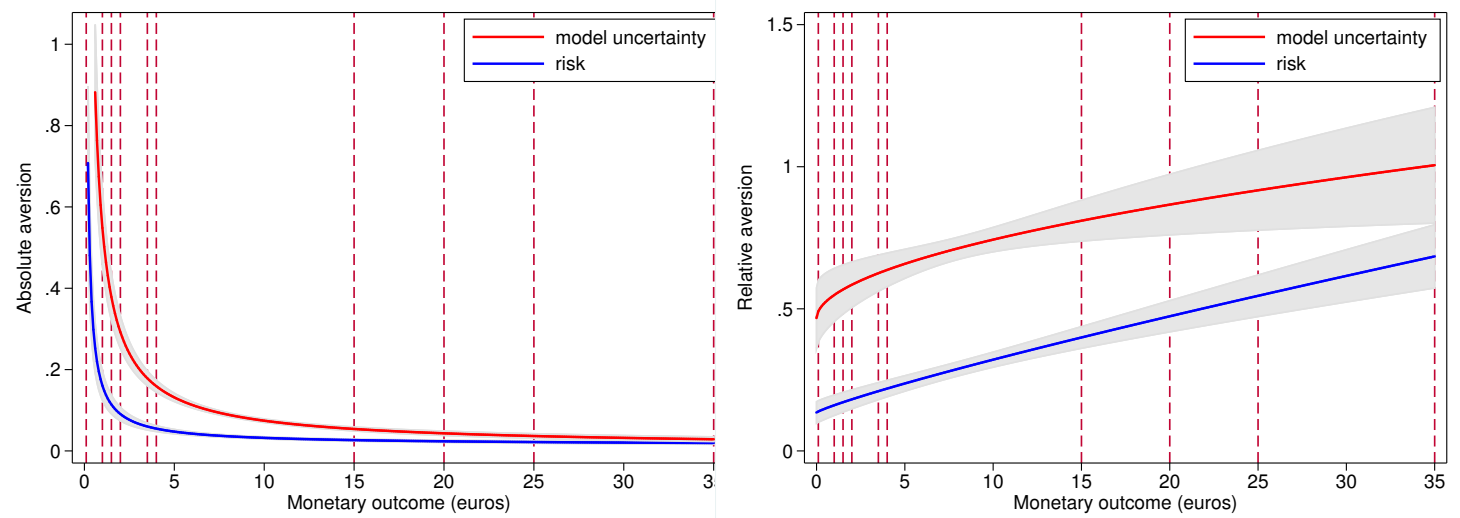

between the attitudes toward objective and subjective probabilities now enable us to quantify the attitude subjects manifest toward ambiguity.

\subsubsection{The implications for ambiguity attitude}

The joint characterization of functions $u$ and $v$ representing the subjects' attitudes toward the different types of uncertainty has an important direct implication for ambiguity aversion. Using the identity $\phi \equiv v \circ u^{-1}$ and the results obtained in the previous section, we now characterize directly the attitude subjects manifest toward ambiguity and compute the parameters of absolute and relative ambiguity aversion (see Appendix A for the detailed analytical computations under the double EP specification). Figure 3 illustrates these parameters. While we observe

Figure 3: Absolute (left) and relative (right) ambiguity aversion obtained with EP function estimates
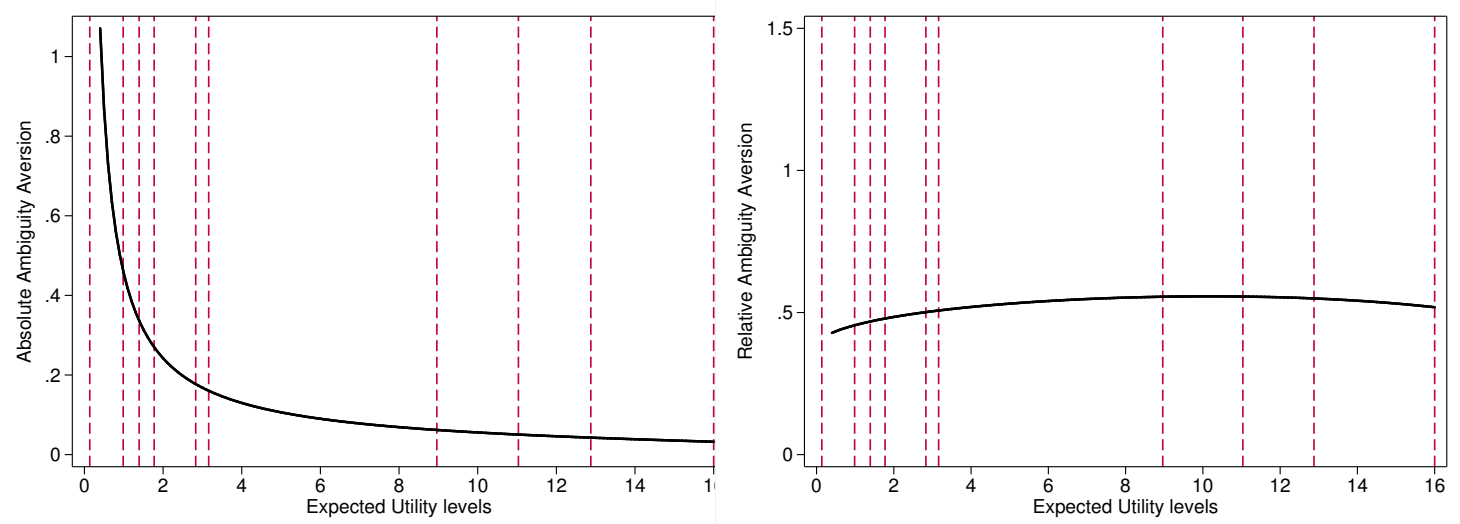

a clearly decreasing trend in the degree of absolute ambiguity aversion, the degree 
of relative ambiguity aversion is fairly constant over the domain considered. As previously mentioned, however, the domain of the ambiguity function $\phi$ is not the same monetary outcome domain as that of $u$ and $v$. Instead, $\phi$ is defined over expected utility levels $U$. In this sense, the vertical dashed lines in Figure 3 represent the levels of utility obtained for the corresponding monetary outcomes in Figure 2, when the utility function $u$ is of the EP type and coefficients are those reported in Table 5.

To assess the robustness of the CRAA result featured in Figure 3, we apply the joint estimation procedure directly to $u$ and $\phi$ (both of which are of the EP type). In other words, we let the ambiguity aversion function be:

$$
\phi(U)=\frac{1-\exp \left(-a_{\phi}(U)^{1-r_{\phi}}\right)}{a_{\phi}},
$$

where $U$ represents the expected utility obtained under a given model $\theta$ (i.e. $U \equiv$ $\left.\hat{p}_{\theta} u(\bar{o})+\left(1-\hat{p}_{\theta}\right) u(\underline{o})\right)$ and $u$ is defined as in equation (13). The estimated results are in the last two columns of Table 5 . In this case, the coefficient $a_{\phi}$ of the EP formulation is not significant at the $5 \%$ level $(p-$ value $=0.062)$. The function describing preferences for ambiguity is therefore instead better represented by a CRAA function. Under this particular specification, we estimate the CRAA parameter to be 0.53. It does not perfectly match the value shown in Figure 3, but this should not be surprising given that the ambiguity functions do not share the same specifications in the two cases. If we instead consider the case of $u$ being CRRA, we also obtain a non-significant coefficient $a_{\phi}(p-$ value $=0.093)$ under the EP specification and estimate the coefficient $r_{\phi}=0.62$ under CRAA. ${ }^{32}$

\section{Conclusion}

Uncertainty is pervasive in both collective and individual decision-making processes. The past few years have witnessed a wealth of studies encompassing multiple academic fields aiming to better formalize the decision process in the face of uncertainty. This body of research investigates, through the development of theoretical frameworks tested through experimental analyses, how individuals integrate available information in the process of decision-making. In particular, scholars have developed multiple decision models to account for attitudes toward ambiguity. They have also adopted these models to explain individuals' behavior

\footnotetext{
${ }^{32}$ In this case, we could have obtained the result directly from the twofold CRRA estimation results provided in Appendix A, given that $\phi$ is of the CRAA type with $r_{\phi}=\frac{r_{v}-r_{u}}{1-r_{u}}$, when both $u$ and $v$ are CRRA (Berger et al., 2017).
} 
in multiple contexts and increasingly applied them to prescribe optimal strategies in the face of uncertainty.

The growing application of ambiguity aversion models calls for the development of experimental efforts enabling a better understanding of the underlying mechanisms at play and a more precise quantification of ambiguity preferences, similar to what has been done in the study of risk. In this paper, we provide new experimental evidence on ambiguity attitudes by means of attitudes toward model uncertainty and risk. In particular, our design enables us to disentangle the role of aleatory and epistemic uncertainty in determining individuals' ambiguity attitudes. We can then quantify, through a joint elicitation procedure, the extent to which ambiguity aversion exists as well as the properties of the ambiguity aversion function in the context of the smooth model.

Two main findings emerge from our analysis. First, we show that subjects tend to be both risk and model uncertainty averse, though they exhibit stronger aversion to model uncertainty than to risk. Following the smooth ambiguity model of choice under uncertainty, we interpret this behavioral characteristic as evidence of ambiguity aversion. Using a joint estimation procedure, we elicit the degree of ambiguity aversion, which we estimate to be twice as large as the degree of risk aversion when considered in relative terms. Second, investigating in more detail attitude toward model uncertainty, we find that model uncertainty aversion is decreasing in wealth when considered in absolute terms and increasing when considered in relative terms. Regarding ambiguity attitude, we find evidence of DAAA and CRAA. These results have several implications for decisions involving aspects of insurance, self-insurance and self-protection in multi-period contexts. Indeed, recent theoretical developments suggest that exhibiting DAAA in the presence of ambiguity will lead to (1) an increase in the insurance coverage rate, (2) raise the optimal level of SI, and (3) favor a higher optimal level of SP (if the degree of disagreement among models is not increasing in the level of effort).

It should, however, be noted that the estimated preferences and parameters we obtained in the context of our experiment may not have predictive power for behavior outside a controlled laboratory environment. Our experimental results should thus be primarily understood as identifying differences between risk and model uncertainty, as well as testing behavioral hypotheses whose relevance may only be fully appreciated within a particular economic framework. Further investigation of individual behaviors in the face of model uncertainty and ambiguity in real-world decisions is therefore warranted. 


\section{Appendix}

\section{A Absolute and relative ambiguity aversion}

When the functions characterizing risk and model uncertainty preferences are both of the expo-power type and are respectively defined as $u(x)=\frac{1-\exp \left(-a_{u} x^{1-r_{u}}\right)}{a_{u}}$ and $v(x)=\frac{1-\exp \left(-a_{v} x^{1-r_{v}}\right)}{a_{v}}$, we can write the ambiguity function $\phi \equiv v \circ u^{-1}$ :

$$
\phi(U)=\frac{1-\exp \left(-a_{v}\left(-\frac{\ln \left(1-a_{u} U\right)}{a_{u}}\right)^{\frac{1-r_{v}}{1-r_{u}}}\right)}{a_{v}},
$$

where $U$ belongs to the space of expected utilities. In this case, we can show that the Arrow-Pratt absolute ambiguity aversion index is:

$$
-\frac{\phi^{\prime \prime}(U)}{\phi^{\prime}(U)}=\frac{a_{v}\left(\frac{1-r_{v}}{1-r_{u}}\right)\left(\frac{-\ln \left(1-a_{u} U\right)}{a_{u}}\right)^{\frac{r_{u}-r_{v}}{1-r_{u}}}+\left(\frac{a_{u}}{\ln \left(1-a_{u} U\right)}\right) \frac{r_{u}-r_{v}}{1-r_{u}}-a_{u}}{1-a_{u} U} .
$$

We can then easily show that in the special case in which $u$ and $v$ are both of the CARA type (i.e. when $r_{u}=r_{v}=0$ ), this index collapses to:

$$
-\frac{\phi^{\prime \prime}(U)}{\phi^{\prime}(U)}=\frac{a_{v}-a_{u}}{1-a_{u} U}
$$

This index is positive whenever $a_{v}>a_{u}$, so that absolute ambiguity aversion results from higher absolute model uncertainty aversion than absolute risk aversion. Similarly, in the special case in which $u$ and $v$ are both of the CRRA type (i.e. when $a_{u}=a_{v}=0$ ), the absolute ambiguity aversion becomes:

$$
-\frac{\phi^{\prime \prime}(U)}{\phi^{\prime}(U)}=\frac{r_{v}-r_{u}}{\left(1-r_{u}\right) U}
$$

and is positive whenever $r_{v}>r_{u}$.

\section{B Robust estimates of risk and model uncer- tainty preferences}

As each of our subjects provided multiple choices in the experiment, we may want to correct for the possible correlation of errors associated with a given subject (which may for example be due to unobserved individual effects). In this 
case, we treat the residuals from the same subject as potentially correlated and make the correction when calculating standard errors of estimates. As Andersen et al. (2008) argued, this procedure allows heteroskedasticity between and within clusters, as well as autocorrelation within clusters, and generalizes the "robust standard errors" approach popular in econometrics. The estimates of risk and model uncertainty aversion with robust standard errors appear in Table B.1.

Table B.1: Robust estimates of risk, model uncertainty and ambiguity preferences

\begin{tabular}{|c|c|c|c|c|}
\hline & \multicolumn{2}{|c|}{$u$} & \multicolumn{2}{|c|}{$v$} \\
\hline & CRRA & $\mathrm{EP}$ & CRRA & $\mathrm{EP}$ \\
\hline$a$ & & $\begin{array}{c}0.0294^{* * *} \\
(0.00398)\end{array}$ & & $\begin{array}{c}0.152^{* * *} \\
(0.0252)\end{array}$ \\
\hline$r$ & $\begin{array}{l}0.279^{* * *} \\
(0.0281)\end{array}$ & $\begin{array}{l}0.135^{* * *} \\
(0.0278)\end{array}$ & $\begin{array}{l}0.738^{* * *} \\
(0.0444)\end{array}$ & $\begin{array}{c}0.467^{* * *} \\
(0.0842)\end{array}$ \\
\hline Noise parameter & $\begin{array}{c}0.103^{* * *} \\
(0.00378)\end{array}$ & $\begin{array}{c}0.105^{* * *} \\
(0.00384)\end{array}$ & $\begin{array}{l}0.0358^{* * *} \\
(0.00492)\end{array}$ & $\begin{array}{l}0.0534^{* * *} \\
(0.00643)\end{array}$ \\
\hline Observations & 5320 & 5320 & 7570 & 75700 \\
\hline Log-likelihood & -1550.3 & -1516.8 & -3682.5 & -3682.1 \\
\hline
\end{tabular}

\section{Sensitivity of model uncertainty aversion}

To gain further insight into the sensitivity of model uncertainty aversion to risk aversion, we used the results from our PL tasks to estimate the degree of relative model uncertainty aversion alone, for values of relative risk aversion ranging from 0 to 0.75 . In this situation the estimation procedure is no longer jointly realized (given the exogenously chosen values of risk aversion considered). In Figure C.1, we present the results of these estimations in the particular case in which both $u$ and $v$ are of the CRRA type. For any value of relative risk aversion, the relative model uncertainty parameter estimated (represented in red) is significantly higher than the 45-degree line (in blue) representing relative risk aversion. In particular, it goes from $r_{v}=0.66$ when $r_{u}=0$ to $r_{v}=0.96$ when $r_{u}=0.75$. We also present in black the degree of relative ambiguity aversion implied by the twofold CRRA specification. Following Berger et al. (2017), the index of relative ambiguity aversion in this situation is given by $r_{\phi}=\frac{r_{v}-r_{u}}{1-r_{u}}$. As we show, $r_{v}$ and $r_{\phi}$ both coincide when risk neutrality is considered (i.e. $r_{u}=0$ ). Following the discussion provided in the main part of the paper, we claim that the level of ambiguity 
Figure C.1: Relative model uncertainty aversion for different values of relative risk aversion when both $u$ and $v$ are of the CRRA type

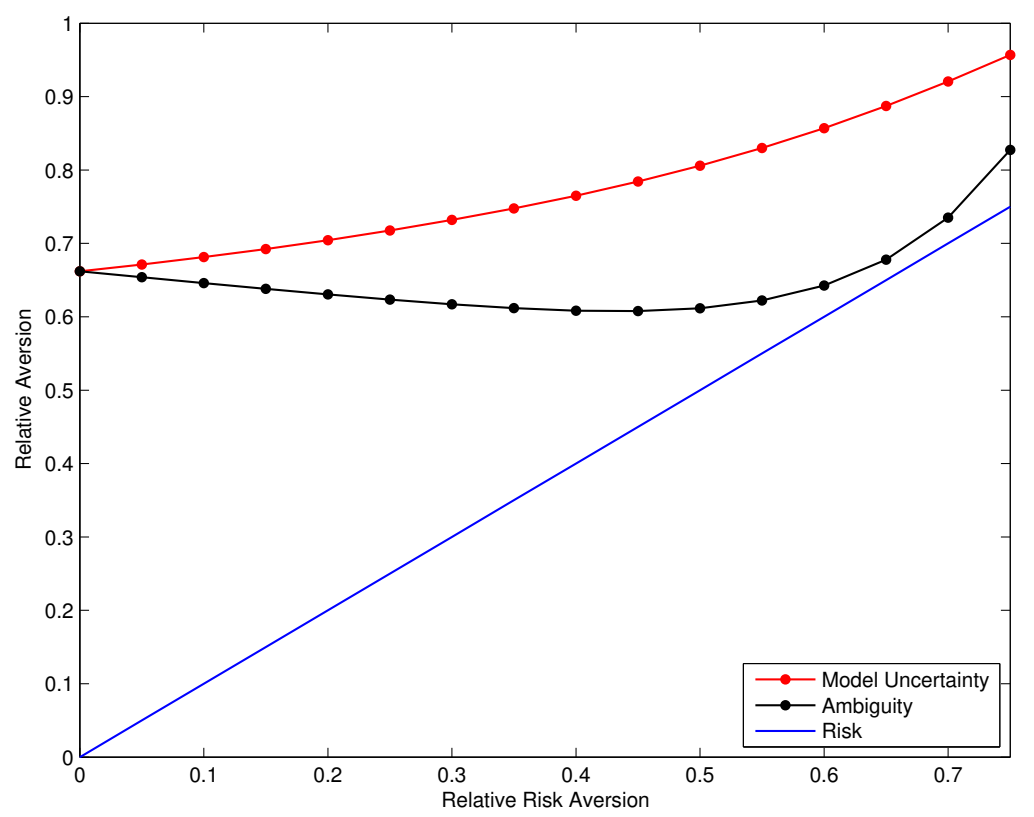

aversion is overestimated when measured in situations of risk neutrality and note that for positive values of $r_{u}$, the index of relative ambiguity aversion is always lower than that of relative model uncertainty aversion. 


\section{References}

Abdellaoui, M., A. Baillon, L. Placido, and P. P. Wakker (2011). The rich domain of uncertainty: Source functions and their experimental implementation. The American Economic Review 101(2), 695-723.

Abdellaoui, M., C. Barrios, and P. P. Wakker (2007). Reconciling introspective utility with revealed preference: Experimental arguments based on prospect theory. Journal of Econometrics 138(1), 356-378.

Adair, J. G., T. W. Dushenko, and R. Lindsay (1985). Ethical regulations and their impact on research practice. American Psychologist 40(1), 59.

Ahn, D., S. Choi, D. Gale, and S. Kariv (2014). Estimating ambiguity aversion in a portfolio choice experiment. Quantitative Economics 5(2), 195-223.

Alary, D., C. Gollier, and N. Treich (2013). The effect of ambiguity aversion on insurance and self-protection. The Economic Journal 123(573), 1188-1202.

Andersen, S., G. W. Harrison, M. I. Lau, and E. E. Rutström (2006). Elicitation using multiple price list formats. Experimental Economics 9(4), 383-405.

Andersen, S., G. W. Harrison, M. I. Lau, and E. E. Rutström (2008). Eliciting risk and time preferences. Econometrica 76(3), 583-618.

Attanasio, O., J. Banks, and S. Tanner (2002, Aug). Asset holding and consumption volatility. Journal of Political Economy 110, 771-792.

Aydogan, I., L. Berger, V. Bosetti, and N. Liu (2018). Three layers of uncertainty and the role of model misspecification: an experiment. IGIER Working Paper 623, Bocconi University.

Baillon, A. and L. Placido (2019). Testing constant absolute and relative ambiguity aversion. Journal of Economic Theory.

Berger, L. (2014). Precautionary saving and the notion of ambiguity prudence. Economics Letters 123(2), 248 - 251.

Berger, L. (2016). The impact of ambiguity and prudence on prevention decisions. Theory and Decision 80(3), 389-409.

Berger, L. (2019). A randomness device to create the conditions of ignorance in the lab. mimeo.

Berger, L. and V. Bosetti (2019). Are policymakers ambiguity averse? The Economic Journal.

Berger, L., J. Emmerling, and M. Tavoni (2017). Managing catastrophic climate risks under model uncertainty aversion. Management Science 63(3), 749-765.

Brunnermeier, M. K. and S. Nagel (2008). Do wealth fluctuations generate timevarying risk aversion? micro-evidence on individuals? asset allocation (digest summary). American Economic Review 98(3), 713-736.

Budescu, D. V., A. K. Rantilla, H.-T. Yu, and T. M. Karelitz (2003). The effects of asymmetry among advisors on the aggregation of their opinions. Organizational Behavior and Human Decision Processes 90(1), 178-194.

Cabantous, L. (2007). Ambiguity aversion in the field of insurance: Insurers? attitude to imprecise and conflicting probability estimates. Theory and Decision 62(3), 219-240.

Cabantous, L., D. Hilton, H. Kunreuther, and E. Michel-Kerjan (2011). Is im- 
precise knowledge better than conflicting expertise? evidence from insurers? decisions in the united states. Journal of Risk and Uncertainty 42(3), 211-232.

Cerreia-Vioglio, S., F. Maccheroni, M. Marinacci, and L. Montrucchio (2013). Classical subjective expected utility. Proceedings of the National Academy of Sciences 110(17), 6754-6759.

Charness, G., U. Gneezy, and A. Imas (2013). Experimental methods: Eliciting risk preferences. Journal of Economic Behavior \& Organization 87, 43-51.

Chetty, R. (2006). A New Method of Estimating Risk Aversion. American Economic Review 96(5), 1821-1834.

Chew, S. H., B. Miao, and S. Zhong (2017). Partial ambiguity. Econometrica $85(4), 1239-1260$.

Chiappori, P.-A. and M. Paiella (2011). Relative risk aversion is constant: Evidence from panel data. Journal of the European Economic Association 9(6), $1021-1052$.

Davis, D. D. and C. A. Holt (1993). Experimental economics. Princeton university press.

Dimmock, S. G., R. Kouwenberg, and P. P. Wakker (2016). Ambiguity attitudes in a large representative sample. Management Science 62(5), 1363-1380.

Dionne, G. and L. Eeckhoudt (1985). Self-insurance, self-protection and increased risk aversion. Economics Letters 17(1-2), 39-42.

Eeckhoudt, L. and C. Gollier (2005). The impact of prudence on optimal prevention. Economic Theory 26(4), 989-994.

Ehrlich, I. and G. Becker (1972). Market insurance, self-insurance, and selfprotection. The Journal of Political Economy 80(4), 623-648.

Ellsberg, D. (1961). Risk, ambiguity, and the Savage axioms. The Quarterly Journal of Economics 75, 643-669.

Epstein, L. G. and Y. Halevy (2018). Ambiguous correlation. The Review of Economic Studies.

Fischbacher, U. (2007). z-tree: Zurich toolbox for ready-made economic experiments. Experimental economics 10(2), 171-178.

Gajdos, T. and J.-C. Vergnaud (2013). Decisions with conflicting and imprecise information. Social Choice and Welfare 41(2), 427-452.

Ghirardato, P. (2004). Defining ambiguity and ambiguity attitude. Uncertainty in economic theory: A collection of essays in honor of David Schmeidler's 65th birthday, 36-45.

Gierlinger, J. and C. Gollier (2008, November). Socially efficient discounting under ambiguity aversion. Technical Report 561, IDEI, Toulouse, Toulouse.

Gilboa, I. and M. Marinacci (2013). Ambiguity and the bayesian paradigm. In Advances in Economics and Econometrics: Theory and Applications, Tenth World Congress of the Econometric Society. D. Acemoglu, M. Arellano, and E. Dekel (Eds.). New York: Cambridge University Press.

Gilboa, I. and D. Schmeidler (1989). Maxmin expected utility with a non-unique prior. Journal of Mathematical Economics 18(2), 141-154.

Grant, S. and B. Polak (2013). Mean-dispersion preferences and constant absolute 
uncertainty aversion. Journal of Economic Theory 148(4), 1361-1398.

Guerdjikova, A. and E. Sciubba (2015). Survival with ambiguity. Journal of Economic Theory 155, 50-94.

Hansen, L. P. (2014). Nobel lecture: Uncertainty outside and inside economic models. Journal of Political Economy 122(5), 945-987.

Hansen, L. P. and M. Marinacci (2016). Ambiguity aversion and model misspecification: An economic perspective. Statistical Science 31, 511-515.

Hansen, L. P. and T. J. Sargent (2001). Robust control and model uncertainty. American Economic Review 91(2), 60-66.

Hardy, G. H., E. M. Wright, D. R. Heath-Brown, and J. H. Silverman (1979). An introduction to the theory of numbers, Volume 4. Clarendon press Oxford.

Harrison, G. W., M. I. Lau, and E. E. Rutström (2007). Estimating risk attitudes in Denmark: A field experiment. The Scandinavian Journal of Economics $109(2), 341-368$.

Harrison, G. W. and E. E. Rutström (2008). Risk aversion in the laboratory. In J. C. Cox and G. W. Harrison (Eds.), Risk Aversion in Experiments (Research in Experimental Economics, Volume 12), Chapter 6, pp. 41-196. Emerald Group Publishing Limited.

Heal, G. and A. Millner (2014). Reflections: Uncertainty and decision making in climate change economics. Review of Environmental Economics and Policy 8(1), 120-137.

Hertwig, R. and A. Ortmann (2008). Deception in experiments: Revisiting the arguments in its defense. Ethics \&5 Behavior 18(1), 59-92.

Holt, C. A. and S. K. Laury (2002). Risk aversion and incentive effects. American economic review 92(5), 1644-1655.

Holt, C. A. and S. K. Laury (2005). Risk aversion and incentive effects: New data without order effects. American Economic Review, 902-904.

IPCC (2014a). Climate Change 2014: Impacts, Adaptation, and Vulnerability. Part A: Global and Sectoral Aspects. Contribution of Working Group II to the Fifth Assessment Report of the Intergovernmental Panel on Climate Change. [Field, CB and Barros, VR and Dokken, DJ and Mach, KJ and Mastrandrea, MD and Bilir, TE and Chatterjee, M and Ebi, KL and Estrada, YO and Genova, $\mathrm{RC}$ and others]. Cambridge, UK/New York, NY: Cambridge University Press.

IPCC (2014b). Climate Change 2014, Mitigation of Climate Change. Contribution of Working Group III to the Fifth Assessment Report of the Intergovernmental Panel on Climate Change. Summary for Policymakers. Working Group III to the Fifth Assessment Report of the Intergovernmental Panel on Climate Change [Edenhofer, O., R. Pichs-Madruga, Y. Sokona, E. Farahani, S. Kadner, K. Seyboth, A. Adler, I. Baum, S. Brunner, P. Eickemeier, B. Kriemann, J. Savolainen, S. Schlömer, C. von Stechow, T. Zwickel and J.C. Minx (eds.)]. Cambridge University Press, Cambridge, United Kingdom and New York, NY, USA.

Jullien, B., B. Salanié, and F. Salanié (1999, June). Should more risk-averse agents exert more effort? The Geneva Risk and Insurance Review 24(1), 19-28.

Klibanoff, P., M. Marinacci, and S. Mukerji (2005). A smooth model of decision 
making under ambiguity. Econometrica 73, 1849-1892.

Krawczyk, M. et al. (2013). Delineating deception in experimental economics: Researchers? and subjects? views. Faculty of Economic Sciences Working Paper, University of Warsaw.

Kriegler, E., J. W. Hall, H. Held, R. Dawson, and H. J. Schellnhuber (2009). Imprecise probability assessment of tipping points in the climate system. Proceedings of the National Academy of Sciences 106(13), 5041-5046.

Lenton, T. M., H. Held, E. Kriegler, J. W. Hall, W. Lucht, S. Rahmstorf, and H. J. Schellnhuber (2008). Tipping elements in the earth's climate system. Proceedings of the National Academy of Sciences 105(6), 1786-1793.

Luce, R. D. (1959). Individual choice behavior. New York: Wiley.

Maccheroni, F., M. Marinacci, and A. Rustichini (2006). Ambiguity aversion, robustness, and the variational representation of preferences. Econometrica 74 (6), 1447-1498.

Marinacci, M. (2015). Model uncertainty. Journal of the European Economic Association 13(6), 1022-1100.

Menges, R. J. (1973). Openness and honesty versus coercion and deception in psychological research. American Psychologist 28(12), 1030.

Saha, A. (1993). Expo-power utility: A "flexible" form for absolute and relative risk aversion. American Journal of Agricultural Economics 75(4), 905-913.

Savage, L. (1954). The Foundations of Statistics. New York: J. Wiley. second revised edition, 1972.

Snow, A. (2011). Ambiguity aversion and the propensities for self-insurance and self-protection. Journal of Risk and Uncertainty 42, 27-43.

von Neumann, J. and O. Morgenstern (1944). Theory of Games and Economic Behavior. Princeton University Press.

Wald, A. (1950). Statistical decision functions. New York: John Wiley \& Sons. 